\title{
UMA APLICAÇÃO DO TDABC - TIME-DRIVEN ACTIVITY BASED COSTING \\ UTILIZANDO A SIMULAÇÃO COMO FERRAMENTA PARA AVALIAÇAO DA QUANTIDADE NECESSÁRIA DE PESSOAL E CONTROLE DE DESPESAS EM UM HOSPITAL MUNICIPAL DO RIO DE JANEIRO
}

\author{
Nélio Achão Filho, M.Sc., Doutorando \\ COPPE/UFRJ - Caixa Postal 68458, CEP 21941-914 - Av. Brigadeiro Trompowski, Ilha do Fundão, Bloco C, \\ RJ - Brasil \\ nachaof@yahoo.com.br
}

Armando Celestino Gonçalves, D.Sc.

UFRJ - Departamento de Engenharia Industrial - Caixa Postal 68458, CEP 21945-970 - Av. Brigadeiro

Trompowski, Ilha do Fundão, Bloco F, RJ - Brasil

armando1964@gmail.com

Osvaldo Luíz Gonçalves Quelhas, D.Sc.

Mestrado Profissional em Sistemas de Gestão/Laboratório de Tecnologia, Gestão de Negócios e Meio Ambiente/UFF - caixa postal 100.175, Cep. 24001-970 - Niterói, RJ - Brasil

quelhas@latec.uff.br

\begin{abstract}
This paper also contributes to improving accessibility to services and quality of emergency medical care is also better distributing the costs involved in a public hospital. Field work is conducted to identifying relevant problems. A simulation model of discrete event is designed to evaluate various scenarios, in order to design the necessary human resources to reduce waiting times in emergency area of the hospital. The relationship between supply and demand of services is studied with emphasis on both the availability of health professionals and real needs of patients. In this context, we inserted a management tool to control costs called TDABC - Time-Driven Activity Based Costing, with the purpose of managing costs more efficiently, and show the strength of it.
\end{abstract}

Key words: Time-Driven Activity Based Costing, Hospital, Management

\section{RESUMO}

O objetivo deste artigo além de contribuir para a melhoria da acessibilidade aos serviços e qualidade do atendimento médico de emergência é também melhor distribuir os custos 
envolvidos no processo em um hospital público. Um trabalho de campo é realizado com vistas a identificar os principais problemas existentes. Um modelo de simulação a eventos discretos é desenvolvido para a avaliação de diversos cenários, com vistas ao dimensionamento dos recursos humanos necessários para a redução do tempo de espera no atendimento de emergência do hospital. A relação entre a oferta de serviços e a demanda é estudada com ênfase tanto na disponibilidade de profissionais de saúde no sistema de admissão quanto nas necessidades reais dos pacientes. Neste contexto, inserimos uma ferramenta gerencial de controle de custos chamada TDABC - Time-Driven Activity Based Costing, com o a finalidade de gerenciar os custos de forma mais eficiente, bem como mostrar a força da mesma.

Palavras-chave: Custo Baseado em Atividades e tempo, Hospital, Gestão.

\section{INTRODUÇÃO}

É de conhecimento geral o estado precário em que se encontram os hospitais públicos do estado do Rio de Janeiro [11]. A situação dos serviços de emergência vem atrelada a esta realidade, agravando-se ainda por causa dos altos custos, falta de infra-estrutura, e por fatores tais como o aumento da violência urbana e rural. Nas emergências de nossos hospitais chegam pacientes a procura de ajuda e muitos não a recebem, seja por falta de informação, de recursos ou de organização. Um sistema eficiente de atendimento de emergência é de interesse particular para a maioria das comunidades, em qualquer lugar do mundo, e um componente crítico do sistema é a resposta de um bom gerenciamento dos serviços [12].

A Pesquisa Operacional (PO) vem sendo utilizada para resolver problemas de administração dos mais variados sistemas organizacionais. No caso específico dos serviços de saúde várias referências sobre a modelagem e a utilização de modelos para a operação de sistemas específicos podem ser encontradas na literatura. Acessibilidade e Qualidade dos Serviços de Saúde foi o tema central de uma Conferência Internacional sobre PO em Serviços de Saúde realizada no Rio de Janeiro. Vários artigos voltados para a realidade brasileira foram publicados recentemente. Vale ressaltar que a busca pela da eficiência como critério básico para a análise dos problemas estudados caracteriza um traço comum a estes trabalhos, que se concentram em transformações restritas ao nível operação dos sistemas de Saúde pública.

A instituição de saúde a ser estudada necessita se reestruturar para, com isso, tentar baixar seus custos operacionais e se tornar mais eficiente. Dessa forma, este estudo busca 
responder a seguinte questão: Como o TDABC pode ser integrado a gestão de Serviços de Saúde?

Para responder esta questão, foram definidos três objetivos. O primeiro objetivo é apresentar uma estrutura conceitual sobre TDABC e simulação. O segundo objetivo consiste em buscar meios para proporcionar a utilização mais adequada dos recursos humanos e materiais, visando melhorar a qualidade da atenção aos pacientes. O terceiro é o desenvolvimento de uma ferramenta de apoio para a tomada de decisão e o estabelecimento de um padrão de qualidade, com base na metodologia TDABC - Time-Driven Activity Based Costing, em atendimento de emergência do Hospital Municipal Miguel Couto - HMMC.

Esta pesquisa se enquadra como explanatória devido à escassez de material bibliográfico a respeito do assunto em questão [6]. Além da busca em documentações, a observação foi outra fonte de evidências ou instrumento de pesquisa utilizado, o que permitiu o conhecimento dos fatos através da visualização dos fatores envolvidos.

O trabalho está organizado da seguinte forma. Depois de feita a introdução, nas seções 2 e 3 apresentam-se os conceitos de simulação e da ferramenta TDABC. Em seguida, na seção 4 evidencia-se um estudo de caso no setor de emergência do Hospital Municipal Miguel Couto - HMMC. Por fim, faz-se uma conclusão do estudo.

\section{O MODELO DE SIMULAÇÃO A EVENTOS DISCRETOS}

A simulação, como técnica de pesquisa operacional, consiste na experimentação numérica com modelos lógico-matemáticos, com o objetivo de estimar parâmetros relativos ao desempenho de sistemas descritivos por estes modelos [11]. A principal vantagem que a simulação tem a oferecer é a sua grande flexibilidade, podendo ser aplicada aos mais variados problemas. Seu emprego restringe-se apenas em função de dificuldades operacionais: na modelagem, na programação, no maior tempo de processamento e na baixa precisão dos seus resultados.

Como o problema estudado é complexo, a formulação é uma tarefa difícil. A construção do modelo lógico representando a formulação do problema é um dos aspectos mais difíceis da modelagem. De fato, entender o problema é o objetivo central do exercício. Nós devemos estar sempre preparados para uma reformulação do problema à medida que obtemos um melhor entendimento das suas características essenciais.

A próxima etapa é converter o modelo lógico num modelo computacional. Este modelo é usado como um instrumento para produzir resultados e conclusões que poderão ser 
implementadas após a validação no mundo real. Em muitas instancias, a função do modelo é funcionar com um meio de comunicação entre aqueles envolvidos no processo de tomada de decisão.

O modelo aqui proposto é baseado em Simulação a Eventos s Discretos - SED. Os elementos de uma SED são entidades ou grupos de entidades tais como pacientes, médicos e leitos e os atributos são propriedades tais como nível de urgência, qualificação e utilização. $O$ estado em que uma entidade se encontra pode ser pensado como um atributo de uma situação corrente. Uma atividade é uma função do sistema na qual uma ou mais entidades permanecem consumindo tempo de simulação. Um evento é uma situação que causa uma mudança no estado do sistema. Neste sentido uma fila é uma lista ordenada de entidades aguardando sua participação numa simples atividade, ou mesmo, num processo mais complexo. A abordagem aqui adotada é orientada ao paciente. Segundo este método, os pacientes são considerados as entidades principais, em torno das quais todas atividades, eventos e processos são organizados. Através da SED, podem-se estudar os efeitos de certas variações no meio ambiente, na organização como um todo, da operação de partes do sistema estudado, com vistas a delinear novas políticas e regras de decisão. A simulação permite avaliar muitas situações que seriam muito difíceis de serem estudadas por modelos de resolução direta. Esta abordagem apresenta assim, inúmeras vantagens com relação a outros métodos de PO.

\section{TIME-DRIVEN ACTIVITY-BASSED COSTING: ABORDAGEM PRÁTICA E MAIS EXATA}

Atualmente já existe uma solução para todos os problemas do ABC convencional. Recentemente, uma nova abordagem denominada Time-Driven Activity_based Costing TDABC [15] surgiu para atender as limitações do ABC. Ela simplifica o processo de custeio, ao eliminar a necessidade de pesquisas e de entrevistas com os empregados, para a alocação dos custos dos recursos às atividades, antes de direcioná-los para os objetos de custos, como pedidos, produtos e clientes.

Este novo modelo atribui os custos dos recursos diretamente aos objetos de custos, por meio de um referencial simples que exige apenas dois conjuntos de estimativas, nenhum dos quais é de difícil obtenção. Primeiro, ele calcula os custos de fornecimento de capacidade dos recursos. Conforme apresentado em [15], por exemplo, veja o caso de um departamento ou processo para o recebimento dos pedidos dos clientes. Nesse passo inicial, o modelo TDABC calcula os custos de todos os recursos - pessoais, supervisão, ocupação, equipamentos e 
tecnologia - fornecidos ao departamento ou processo. Esse custo total é dividido pela capacidade - o tempo disponível dos empregados que efetivamente executam o trabalho - do departamento, de modo a determinar a taxa do custo da capacidade.

Segundo, o TDABC usa a taxa do custo da capacidade para distribuir os custos dos recursos departamentais entre os objetos de custos, estimando a demanda de capacidade de recursos por cada objeto de custos (tipicamente tempo, o qual determinou a denominação da abordagem). Prosseguindo em nosso exemplo do departamento de pedidos de clientes, o modelo exige apenas uma estimativa do tempo necessária para processar determinado pedido de cliente. Mas o TDABC não exige que todos os pedidos de clientes sejam iguais. Ele permite que a estimativa de tempo varie em função das características especificas de cada caso, tais como grau de automatização do pedido, prazo de entrega, pedidos expedidos, pedidos internacionais, fragilidade e perecibilidade do produto, ou pedido de novo cliente sem disponibilidade de cadastro de crédito existente

$\mathrm{O}$ modelo TDABC simula os processos vigentes para executar o trabalho em toda empresa. Portanto, é capaz de refletir muito mais variação e complexidade que o modelo ABC convencional, sem criar demanda explosiva de estimativas, de armazenamento e de capacidade de processamento de dados. Usando o modelo TDABC a empresa é capaz de abarcar a complexidade, em vez de ser forçada a adotar modelos ABC simplificados e inexatos para refletir a complexidade dos negócios.

Estudos realizados aplicando este modelo enfatizaram-se, principalmente, os benefícios trazidos empiricamente pelo TDABC nas organizações analisadas [6; 11].

\subsection{EQUAÇÕES DE TEMPO}

A abordagem do TDABC requer estimativa de somente dois parâmetros: o custo unitário da capacidade de recursos fornecidos e a unidade de tempo exigida para a executar uma transação ou atividade [15]. Ele incorpora com facilidade variações nas demandas de tempo em diferentes tipos de transações. Ela não exige as premissas simplificadoras, necessárias até aqui, de que todos os pedidos ou transações sejam iguais e exijam o mesmo tempo de processamento. Podemos admitir que as unidades de tempo estimadas no modelo TDABC variem com base nas características do pedido e da atividade.

Em artigo realizado em uma empresa por encomenda, concluiu-se que a identificação e a definição das equações de tempo são as principais dificuldades para a aplicação do 
TDABC, devido às atividades apresentarem uma grande imprevisibilidade, tanto com relação ao tempo de execução quanto à intensidade do consumo de recursos.

A abordagem Time-Driven estima a demanda de recursos do departamento por meio de uma equação simples:

Equação de tempo $=\beta_{0}+\sum_{i=1}^{n} \beta_{i} \mathrm{X}_{i}=\beta_{0}+\beta_{1} \mathrm{X}_{1}+\ldots+\beta_{n} \mathrm{X}_{n}$

Embora aparentemente complexas, envolvendo grande volume de dados, as equações de tempo, em geral, são muito simples de implementar, uma vez que, os sistemas integrados de gestão de muitas empresas já dispõem de informações sobre pedidos, embalagem, distribuição e outras características. Esses dados específicos sobre pedidos e transações criam condições para que as demandas de tempo específicas de qualquer pedido sejam calculadas com rapidez, por meio de um algoritmo simples que testa a existência de cada atributo que afeta o consumo de recursos.

Os modelo TDABC se expandem linearmente, em função de variações, mediante o acréscimo de termos às equações de tempo. As equações de tempo no modelo TDABC também oferecem aos gestores a possibilidade de simular o futuro.

\subsection{ATUALIZAÇÃO DO MODELO}

Os gestores podem atualizar com facilidade o modelo TDABC para refletir mudanças em suas condições operacionais. Eles não precisam entrevistar novamente o pessoal quando o departamento passa a executar mais atividades. Simplesmente estimam as unidades de tempo necessárias para cada nova atividade identificada. Os gestores podem incorporar o efeito de pedido complexo versus pedidos simples, estimando a necessidade de aumento de unidade de tempo quando se precisa lidar com uma transação complexa.

\section{ESTUDO DE CASO: HOSPITAL MUNICIPAL MIGUEL COUTO}

O HMMC é referência em trauma e cobre uma área extensa do município do Rio de Janeiro. O Hospital possui 389 leitos, 1750 funcionários e é uma unidade de saúde caracterizada pelo atendimento secundário e terciário, atendendo a mais de 400.000 pacientes 
por ano. O Hospital conquistou, ao longo dos anos, respeito e admiração do público, pelo excelente trabalho de seus profissionais, em particular na área de emergência.

Os serviços disponíveis são: Triagem hospitalar; Emergência; Maternidade; Laboratório; Radiologia; Ambulatórios; Serviços clínicos e Cirurgias. As rotinas operacionais do HMMC são concentradas nas atividades relacionadas com assistência ambulatorial em suas unidades de emergência e ambulatório e, não obstante, nas atividades relacionadas com os pacientes internos.

O conjunto das operações de pacientes internos pode ser classificado [4], como: Internação eletiva de paciente de uma unidade médico assistencial qualquer; Internação de urgência/emergência; Internação em outro hospital próprio, de co-gestão, de convênio ou contratado; Transferência de pacientes em leitos de uma mesma clínica ou interclínicas e Procedimentos relacionados com a alta de pacientes.

\section{DEFINIÇÃO DO ESTUDO}

\subsection{O MODELO DE ATENÇÃO AO PACIENTE}

A elaboração de um modelo de simulação deve começar pela formulação do problema e por uma definição clara dos objetivos do experimento. A formulação é um processo onde desenvolvemos o modelo conceitual do problema, ou seja, aquilo que é imaginado por quem modela. A modelagem é baseada no fluxo de admissão do paciente, que é considerado como a entidade mais importante, e em torno da qual, uma série de atividades é realizada. O fluxo do paciente é considerado como um ciclo fechado de filas e atividades. Uma forma sintética do funcionamento do modelo é considerar o paciente como a entidade principal e o seu fluxo.

A cada atividade, uns conjuntos de entidades interagem, sob certas condições, gerando eventos que modificam o estado das filas. O comportamento dinâmico do sistema é avaliado no experimento de simulação. Os dados utilizados na simulação foram estimados com base no relatório dos índices de atividades do HMMC, referente ao ano de 2002. Os resultados revelam que a chegada dos pacientes segue uma distribuição exponencial: A cada dois minutos chega um paciente. A taxa de serviço segue uma distribuição exponencial. Após a entrada, os pacientes têm o primeiro contato com o guarda, e são encaminhados para o atendimento de emergência, recepção ou para a triagem.

O critério de escolha é atender primeiro o caso com mais urgência. Para pacientes em igualdade de urgência, que seu estado permita esperar, o critério de atendimento é: o primeiro 
a chegar é o primeiro a ser atendido. Evidentemente, devido à demanda excessiva e desorganização, esses critérios falham. Note que quem administra a porta de entrada, sem formação para isso, é o guarda. Nas Figuras 1 e 2 apresentamos a modelagem de dois cenários distintos; a situação atual, congestionada, uma proposta de organização.

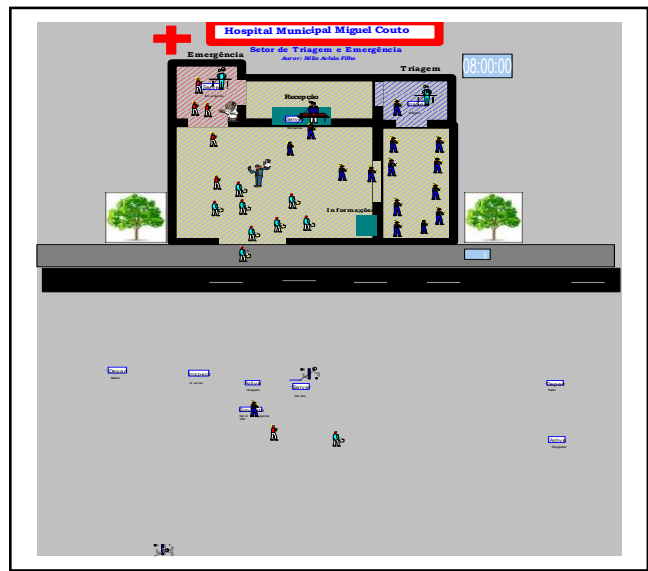

Figura 1- Situação atual do hospital

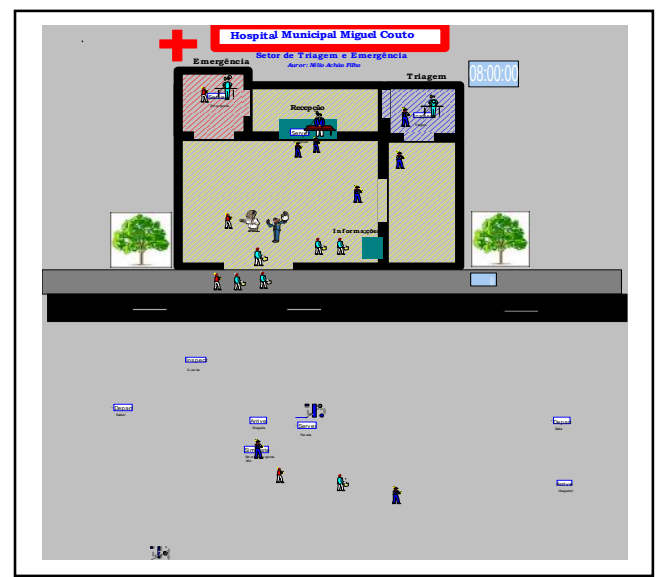

Figura 2 - Proposta de organização

\subsection{A QUALIDADE NA ATENÇÃO AOS PACIENTES}

A atenção aos pacientes é desenvolvida por uma equipe que inclui médicos, enfermeiras, auxiliares de enfermagem, atendentes, etc. As equipes oferecem atenção a todos os pacientes na sala. Equipes interagem diretamente com o paciente e podem dar atenção simultaneamente a mais de um paciente se for preciso. Além disso, alguns membros desta equipe, podem colaborar com outras equipes na atenção a outros pacientes. Leva-se em consideração também características próprias de cada especialidade.

Neste artigo, estamos avaliando a qualidade do processo da atenção e da estrutura, especificamente, a ordem dos acontecimentos dos processos, relativos a atenção ao paciente, e o aporte de todos os profissionais de saúde, no tempo requerido e na quantidade precisa. $\mathrm{O}$ estado da espera dos pacientes é analisado de forma geral. A Tabela 2 mostra, como exemplo, os escores utilizados para as avaliações apresentamos as avaliações relativas do sistema.

\begin{tabular}{|c|c|}
\hline Tempo (min) & Avaliação da Qualidade (Scores) \\
\hline$(0-1]$ & 10 \\
\hline$(1-2]$ & 10 \\
\hline$(2-5]$ & 9 \\
\hline$(5-15]$ & 5 \\
\hline$(15-30]$ & 2 \\
\hline
\end{tabular}




\begin{tabular}{|c|c|}
\hline$(30-60]$ & 1 \\
\hline$>60$ & 0 \\
\hline
\end{tabular}

Tabela 2: Escores para avaliação da qualidade de atenção

\subsection{ANÁLISE DE RESULTADOS E SENSIBILIDADE DO MODELO}

Uma série de experimentos foi realizada e diversas configurações de equipe médica foram estudadas. A configuração: atual do hospital é: (a) 4 médicos na triagem; (b) 2 médicos na emergência; e (c) 2 atendentes na recepção. Para a especialidade escolhida, o seguinte experimento foi proposto: (a) Variar o número de médicos; (b) Variar a taxa de chegada; e (c) Avaliar o processo de encaminhamento;

Realizamos várias rodadas de simulação partindo da situação atual e variando a quantidade de médicos da triagem e da emergência e mantendo fixa a quantidade de atendentes na recepção. O período de 6 horas foi escolhido tendo em vista a distribuição dos três turnos do hospital. Duas situações distintas são exploradas, mas só uma será mostrada na Tabela 3. A primeira hipótese considera fixo, na triagem, o número de médicos igual a 9 e o número de atendentes na recepção igual a 2 , variando-se apenas a quantidade de médicos na emergência de 2 até 8 .

O comportamento o tempo de espera na fila é observado. Na segunda situação, foi mantida constante, na triagem, a quantidade de médicos igual a 10, e o número de atendentes, na recepção igual a 2, variando apenas a quantidade de médicos na emergência, só que agora, entre 2 e 7. Maiores detalhes sobre este experimento podem ser encontrados em outro estudo [4].

A tabela 3, a seguir fornece o resultado final da avaliação, tendo em vista os tempos médios de espera na fila. O que nos interessa, na verdade é avaliar os critérios, préestabelecidos, de qualidade. Os scores estabelecidos nas tabelas de avaliação da formulação do modelo são então utilizados.

\begin{tabular}{|c|c|c|c|c|c|c|}
\hline Função / Quantidade & Setor & $\begin{array}{c}\text { Taxa de } \\
\text { Ocupação }\end{array}$ & $\begin{array}{c}\text { Frequiência } \\
\text { Observações }\end{array}$ & $\begin{array}{c}\text { Tempo médio } \\
\text { na fila }\end{array}$ & $\begin{array}{c}\text { Avaliação da } \\
\text { Qualidade } \\
\text { (Scores) }\end{array}$ & Média (Scores) \\
\hline Atendente - 2 & Recepção & 0,80 & 145 & 0,15 & 10 & \\
\hline Médico -10 & Triagem & 5,84 & 142 & 0,05 & 10 & 10 \\
\hline Médico -5 & Emergência & 2,63 & 25 & 1,25 & 10 & \\
\hline Atendente - 2 & Recepção & 0,80 & 145 & 0,15 & 10 & \\
\hline Médico $\quad-10$ & Triagem & 5,84 & 142 & 0,05 & 10 & 10 \\
\hline Médico $\quad-6$ & Emergência & 2,63 & 25 & 0,34 & 10 & \\
\hline Atendente - 2 & Recepção & 0,80 & 145 & 0,15 & 10 & \\
\hline
\end{tabular}




\begin{tabular}{|ll|l|c|c|c|c|c|}
\hline Médico -10 & Triagem & 5,84 & 142 & 0,05 & 10 & 10 \\
\hline Médico -7 & Emergência & 2,63 & 25 & 0,00 & 10 & \\
\hline
\end{tabular}

Tabela 3- Avaliações da qualidade do tempo de espera nas filas

A Tabela 3 considera o grau de aproximação com os critérios de qualidade préestabelecidos. Mostramos apenas, a título de exemplo, os valores referentes às médias finais iguais a 10, ou seja, os menores tempos de espera para atendimento ao paciente, que é a situação mais favorável do nosso experimento. Podemos verificar que para a hipótese 2 atendentes na recepção, com tempo de atendimento médio de 2 minutos, encontramos 145 pacientes esperando menos que 1 minuto para serem atendidos. Para a hipótese de 10 médicos na triagem com tempo de atendimento médio de 15 minutos cada um, tem que 142 pacientes esperam menos de 1 minuto para serem atendidos. Agora, podemos verificar que para a hipótese de 7 médicos na emergência, com tempo de atendimento médio de 40 minutos cada um, 25 pacientes esperam muito pouco tempo para serem atendidos.

Procedendo assim, através de uma série de experimentos podemos chegar ao resultado desejado, ou seja: a configuração adequada da equipe que possa atender aos pacientes de emergência da melhor forma possível e que não haja espera por parte dos pacientes de emergência. $\mathrm{O}$ modelo de simulação pode ser muito útil para avaliar a qualidade em três níveis: (a) Configuração da equipe; (b) Seqüência correta dos procedimentos médicos; (c) Redução do tempo de espera.

A configuração da equipe é feita em termos do número de profissionais. Os procedimentos são diretamente relacionados com o grau de urgência e o tempo de espera está intimamente associado com os outros níveis. A complexidade do problema é aparente. A titulo de ilustração mostramos algumas situações que ocorrem na análise deste problema complexo. A questão do tempo de espera para o atendimento na triagem requer uma reflexão, profunda, pois este atendimento básico é um primeiro contato do paciente com o sistema. Uma boa triagem serve para qualificar a demanda e evitar uma congestão do serviço.

Podemos observar que a situação ideal seria ter 10 médicos na triagem. Nota-se ainda que houve uma grande variação no tempo de espera quando o número de médicos variou de 4 para 5. O mesmo acontece de 6 para 7 médicos. Esta informação é importante, mas deve ser ainda avaliar uma série de outras hipóteses antes de uma decisão. As variações da demanda, o tempo de atendimento, o tempo ocioso dos médicos, o custo e o fluxo de atendimento devem também ser avaliados. 
Este gráfico foi produzido para o caso em que o tempo estabelecido para o atendimento é de 15 minutos. Uma análise interessante é avaliar qual o número adequado de médicos para o setor de emergência, que reduzirá o tempo de espera para os pacientes oriundos da triagem médica. $\mathrm{O}$ atendimento de emergência normalmente ocorre após a triagem. Deseja-se avaliar o tempo de espera na fila do atendimento de emergência propriamente dito.

A Figura 2 considera a configuração de médicos na emergência para o caso mais favorável da situação anterior, que é o de se dispor de 10 médicos na triagem. A melhor situação ocorre com 7 médicos na emergência. Um outro fato interessante, é que ao se aumentar o número de médicos de 2 para 3, houve uma variação significante no tempo de espera. O mesmo aconteceu ao se aumentar o número de médicos de 3 para 4. Isso pode estar relacionado à própria taxa de chegada aliada com o tempo estabelecido para o atendimento que é de 40 minutos. A percentagem de pacientes são encaminhados para a emergência a partir da triagem é conhecida. Entretanto, a demanda é muito grande para todo o serviço. É evidente que essa avaliação foi feita levando-se em consideração apenas uma visão parcial do problema. Um estudo bem mais detalhado que foge ao escopo deste trabalho.

Neste estudo preliminar, concluímos que, para uma demanda semelhante á atual, necessitamos dimensionar uma equipe médica com 17 médicos, sendo 10 para a triagem e 7 para a emergência. A quantidade de 2 atendentes na recepção nos parece adequada. Vale ressaltar, que estamos considerando aqui apenas uma especialidade médica. Um trabalho criterioso para a avaliação da demanda e cálculo da taxa de chegada de pacientes deverá ser feita, para que se possa dimensionar e alocar os recursos disponíveis de maneira mais precisa.

Diante do exposto, contudo, os responsáveis pelo hospital devem levar em consideração, antes de uma análise para dimensionamento dos processos, itens importantes como disponibilidade de recursos financeiros, materiais e humanos, entre outros. Após a análise da quantidade dos recursos necessários vamos introduzir alguns conceitos básicos sobre a ferramenta de custos que vamos utilizar na elaboração do trabalho. Vamos utilizar os resultados analisados da simulação para dimensionar a metodologia TDABC conforme visto a seguir.

\subsection{APLICAÇAO DO TDABC}

A primeira decisão tomada foi relativa ao alcance do trabalho a ser realizado, em termos de estrutura organizacional. Após a introdução dos conceitos do TDABC serão 
definidos alguns itens para custeio. Para facilitar a aplicação do método foram escolhidas 5 atividades nas quais estabelecemos a quantidade de horas consumidas no setor de emergência. Em seguida, faremos algumas suposições tais como: quantidades da atividade, custo da capacidade fornecida com base nos relatórios gerenciais da empresa, horas fictícias gastas por atividade com base em informações do próprio setor, numero de funcionários, taxa de trabalho e horas não produtivas. No tocante a cooperação dos funcionários, salva algumas exceções, não foi possível a convocação destes a colaborar com o estudo. Por isso, o estudo foi baseado nas propostas teóricas discutidas no início deste trabalho com algumas adaptações dos modelos propostos por [3], modelos esses que melhor atendem ao estudo.

Esse modelo simplificado pressupõe a criação de um grupo de trabalho na empresa estudada, formado por pessoas envolvidas no processo de planejamento e controle de e custos. Este grupo utilizará seus conhecimentos e experiências adquiridas, para fornecer dados, informações e análises, sobre a adequada avaliação das horas aplicadas a cada atividade. A empresa tem um custo de capacidade fornecida trimestral da ordem de \$ 400.000,00 (Dado fictício baseado em relatórios gerenciais) para alocar. Estabelecemos os minutos consumidos por atividade e a quantidade total de minutos multiplicando-se pela quantidade de vezes que estas são realizadas no período, no caso 326.400 minutos conforme Tabela 4.

\subsubsection{TIME-DRIVEN ACTIVITY-BASED COSTING}

O custo da capacidade fornecida é, neste caso, $\$ 400.000$ por trimestre. Para estimar a capacidade prática, a equipe de TDABC identifica a quantidade de recursos (tipicamente, pessoas e equipamentos) que realmente executam o trabalho. Presuma que o departamento utiliza 17 funcionários de linha de frente, sendo que não foram incluídos supervisores ou pessoal de apoio. Cada funcionário de linha de frente trabalha, em media, 20 dias por mês (60 dias por trimestre) e é remunerado com base em 6 horas de trabalho por dia. Portanto, cada empregado está presente no local de trabalho em media por cerca de 360 horas, ou 21.400 minutos por trimestre.

No entanto, nem todo o tempo remunerado é aproveitado em trabalho produtivo. Os empregados do setor de emergência passam, aproximadamente, 40 minutos por dia em intervalos dos mais diversos. Assim, a capacidade prática de cada empregado é cerca de 19.200 minutos por trimestre (320 minutos por dia, multiplicados por 60 dias por trimestre). Com 17 funcionários de linha de frente, a capacidade prática do departamento perfaz 326.400 minutos. A taxa de custo (por minuto) de capacidade de fornecimento, primeiramente 
estimada para o modelo TDBAC, pode agora ser calculada: Taxa do custo da capacidade $=\$$ 400.000 / 326.400 minutos. A estimativa da capacidade prática de um funcionário ou de uma parte do equipamento deve ser direta. Calcule quantos dias por mês, em média, os empregados e as máquinas trabalham, e em quantas horas ou em quantos minutos por dia os funcionários ou os equipamentos realmente estão disponíveis para a realização do trabalho, depois de subtrair os intervalos para repouso, treinamento, reuniões, manutenção e quaisquer outros intervalos. Os cálculos não precisam ser absolutamente exatos. Erros de alguns pontos percentuais raramente serão fatais e erros vultosos serão detectados através de inesperadas faltas ou excessos de capacidade. A segunda estimativa exigida pelo modelo TDABC é a capacidade necessária - nesse e na maioria dos casos - tempo - para executar cada transação. $\mathrm{O}$ ABC convencional usa um direcionador de transação sempre que uma atividade - como configurar máquina, emitir pedido de compra, ou processar solicitações de clientes - consome mais ou menos a mesma quantidade de tempo. O TDABC, em vez de usar esses direcionadores de transação, simplesmente requer que a equipe do projeto estime o tempo necessário para executar cada uma dessas atividades transacionais.

\begin{tabular}{|c|c|c|c|c|c|}
\hline 2 & $\begin{array}{l}\text { Número de funcionários diretos: } \\
\text { Taxa de tabalho (h/dia): }\end{array}$ & $\begin{array}{r}17 \\
6\end{array}$ & & & \\
\hline 3 & Custo capacidade fornecida: & $\begin{array}{r}6 \\
400.000,00\end{array}$ & & & \\
\hline 4 & Periodo considerado (3 meses): & $400.000,00$ & & & \\
\hline 5 & Horas não produtivas (min/dia/empregado): & 40 & & & \\
\hline 6 & Horas não produtivas (min/60dias/empregado): & 2.400 & & & \\
\hline 7 & Tempo total ( $\mathrm{min} / 60 d i a s /$ mpregado): & 21.600 & & & \\
\hline 8 & Capacidade prática: & 19.200 & & & \\
\hline \multirow{3}{*}{10} & Capacidade prática dos recursos fornecidos: & 326.400 & & & \\
\hline & Taxa do custo da capacidade $=$ & 1,2255 & & & \\
\hline & Atividades / tempo & Horas & Min & \multirow[b]{8}{*}{$\begin{array}{c}\text { Quantidade de } \\
\text { direcionadores } \\
\text { de custo }\end{array}$} & \multirow[b]{8}{*}{$\begin{array}{c}\text { Taxa do } \\
\text { direcionador de } \\
\text { custo }(\$)\end{array}$} \\
\hline 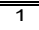 & "Atividade de receber paciente & 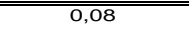 & 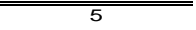 & & \\
\hline 2 & Atividades de utilizar equiptos especificos & 0,50 & 30 & & \\
\hline 3 & Atividades de executar procedimento especif. & 0,42 & 25 & & \\
\hline 4 & Atividades de receitar & 0,17 & 10 & & \\
\hline \multirow[t]{3}{*}{5} & Atividades de encaminhar para outro setor/local & 0,25 & 15 & & \\
\hline & \multicolumn{3}{|c|}{ ABC Convencional } & & \\
\hline & Atividades & $\begin{array}{c}\text { Tempo } \\
\text { consumido (\%) }\end{array}$ & $\begin{array}{l}\text { Custo Atribuido } \\
\text { (\$) }\end{array}$ & & \\
\hline 1 & Atividade de receber paciente & 5,9 & $23.529,41$ & 3600 & 6,54 \\
\hline 2 & Atividades de utilizar equiptos especificos & 35,3 & $141.176,47$ & 3600 & 39,22 \\
\hline 3 & Atividades de executar procedimento especif. & 29,4 & $117.647,06$ & 3600 & 32,68 \\
\hline 4 & Atividades de receitar & 11,8 & $47.058,82$ & 3600 & 13,07 \\
\hline \multirow[t]{3}{*}{5} & Atividades de encaminhar para outro setor/local & 17,6 & $70.588,24$ & 300 & 235,29 \\
\hline & Total & 100 & $400.000,00$ & & \\
\hline & Atividades & $\begin{array}{r}\text { TDABC } \\
\text { Unidade de } \\
\text { tempo (min) }\end{array}$ & Taxa por min & & \\
\hline 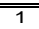 & Atividade de receber paciente & 5 & 6,13 & & \\
\hline 2 & Atividades de utilizar equiptos especificos & 30 & 36,76 & & \\
\hline 3 & Atividades de executar procedimento especif. & 25 & 30,64 & & \\
\hline 4 & Atividades de receitar & 10 & 12,25 & & \\
\hline 5 & Atividades de encaminhar para outro setor/local & 15 & 18,38 & & \\
\hline
\end{tabular}

\begin{tabular}{|c|c|c|c|c|c|c|}
\hline & Atividades & $\begin{array}{r}\text { TDABC } \\
\text { Quantidade } \\
\end{array}$ & $\begin{array}{l}\text { Unidade de } \\
\text { tempo (min) }\end{array}$ & Tempo total & $\begin{array}{c}\text { Custo unitário } \\
(\$ / \mathrm{min})\end{array}$ & $\begin{array}{c}\text { Custo total } \\
\text { atribuido (\$) }\end{array}$ \\
\hline 1 & Atividade de receber paciente & 3.600 & $\frac{5}{5}$ & 18.000 & 6,13 & $22.058,82$ \\
\hline 2 & Atividades de utilizar equiptos especificos & 3.600 & 30 & 108.000 & 36,76 & $132.352,94$ \\
\hline 3 & Atividades de executar procedimento especif. & 3.600 & 25 & 90.000 & 30,64 & $110.294,12$ \\
\hline 4 & Atividades de receitar & 3.600 & 10 & 36.000 & 12,25 & $44.117,65$ \\
\hline 5 & Atividades de encaminhar para outro setor/local & 300 & 15 & 4.500 & 18,38 & $5.514,71$ \\
\hline 6 & Capacidade utilizada & & & 256.500 & & $314.338,24$ \\
\hline 7 & Capacidade não-utilizada $(21,42 \%)$ & & & 69.900 & & $85.661,76$ \\
\hline & Total & & & 326.400 & & $400.000,00$ \\
\hline
\end{tabular}

Tabela 4: Aplicação do TDABC 
As estimativas de tempo podem ser obtidas através de observação direta por entrevistas. Como no caso da estimativa da capacidade prática, a exatidão não é critica, bastando uma acuracidade aproximada. E, ao contrário das porcentagens que os empregados estimam subjetivamente no modelo ABC convencional, as estimativas do consumo de capacidade no modelo Time-Driven podem ser imediatamente observadas e validadas. Sendo assim, suponha que a equipe do TDABC estime os seguintes tempos médios unitários para as oito atividades relacionadas ao processo: (a) Receber paciente: 5 minutos; (b) Utilizar equipamentos específicos: 30 minutos; (c) Executar procedimento específico: 25 minutos; (d) Receitar: 10 minutos; e (e) Encaminhar para outro setor / local: 15 minutos.

A equipe agora simplesmente calcula as taxas dos direcionadores de custo para os oito tipos de atividades executadas no setor de emergência, multiplicando a taxa do custo da capacidade pela unidade de tempo estimada de cada atividade.

\section{AVALIAÇÃO DO ESTUDO E SUGESTÕES}

A análise revela que apenas cerca de $78,58 \%$ da capacidade prática dos recursos fornecidos durante o período foram usados em trabalho produtivo. Conseqüentemente, apenas $78,58 \%$ das despesas totais de $\$ 400.000,00$ são atribuídas ao processo durante esse período. A Tabela 4 mostra o tempo necessário para executar as cinco atividades definidas, assim como, os custos dos recursos. Também enfatiza a diferença entre capacidade fornecida e capacidade utilizada. Os gestores podem analisar o custo da capacidade não-utilizada e pensar em medidas para determinar se é possível reduzir os custos do fornecimento de recursos nãoutilizados em períodos subseqüentes, e como fazer isso. Em vez de reduzir a capacidade nãoutilizada no presente, os gestores podem optar por reservar essa capacidade para crescimento futuro.

No caso estudado, as análises poderiam ser complementadas após mapeamento de todas as outras áreas realizadas pelo hospital, as quais não foram consideradas por questões de simplicidade do estudo. Desta forma, seria possível também irmos ao detalhe de cada processo, entretanto isso levaria mais tempo e consumiria mais recursos. A adequação do desenvolvimento da ferramenta parece ter sido satisfatória tendo em vista o grau de abrangência das considerações pré-estabelecidas. 


\section{CONCLUSÃO}

Os hospitais públicos do município do Rio de Janeiro vêm passando por várias transformações ao longo dos anos. Novas tecnologias de gerenciamento estão surgindo e, com elas, maiores possibilidades de melhoria nas estruturas organizacionais, visando cada vez mais satisfazer as necessidades dos pacientes que sempre foram meros coadjuvantes nesse processo. È de conhecimento geral existe uma série de problemas administrativos que poderiam ser facilmente resolvidos com o auxilio da PO. Nosso objetivo foi aplicar um método de análise que permite a avaliação da performance do setor de emergência de um hospital público. O fluxo de admissão dos pacientes é estudado e um modelo de simulação é utilizado para avaliar a demanda e a oferta de serviços, tomando como base a porta de entrada do hospital, e os serviços de triagem e atenção primária de emergência.

Por meio da simulação, várias rodadas do modelo foram realizadas, variando-se a demanda, as quantidades de membros da equipe e outros parâmetros de interesse para a avaliação da performance do sistema. O estudo revelou informações relevantes sobre a performance do sistema, como a quantidade de profissionais necessária para uma redução do tempo de espera, e o número adequado de profissionais de cada categoria, para que o serviço seja mais eficiente e eficaz. O modelo aponta para a integração entre os serviços e uma avaliação neste sentido é realizada para uma das situações favoráveis.

A partir de uma tabela de scores, pré-definidos pelas equipes médicas, foi feita a avaliação da qualidade dos serviços, redução do tempo de espera na fila para atendimento, com vistas a avaliar as condições favoráveis de funcionamento na situação atual. A metodologia fornece informações relevantes para que os decisores possam definir seu nível de precisão e viabilidade; levando-se em consideração, sobretudo, os custos. Neste sentido, apresentamos e aplicamos uma ferramenta gerencial de controle custos, chamada TDABC Time-Driven Activity Based Costing, no intuito de aumentar a eficiência no tocante a melhor distribuição dos custos. Por fim, nossa contribuição foi além de apontar algumas deficiências com relação à quantidade de médicos disponíveis na triagem e na emergência, para a demandas duas taxas de chegada adotadas uma metodologia de distribuição de custos mais eficiente para controle gerencial. A metodologia demonstrou sua importância; fornecendo dados básicos, para que o hospital avalie suas estruturas organizacionais, físicas e de recursos humanos; e assim, possa dimensionar melhor seu processo e sua estrutura de custos. 


\section{REFERÊNCIAS BIBLIOGRÁFICAS}

[1] ACHÃO FILHO, N., ANACLETO, M.A. \& QUELHAS, O.L.G. - "Critérios para Aplicação de ABC (Activity Based Costing) e BSC (balanced Scorecard) na Indústria Naval" - SPOLM: Simpósio de Pesquisa Operacional e Logística da Marinha, Rio de Janeiro, 2008.

[2] ACHÃO FILHO, N., ANACLETO, M.A. \& DE OLIVEIRA, M.J.F. - "A simulação como método de avaliação da qualidade de atendimento e fonte de dados para o BSC na emergência do Hospital Municipal Miguel Couto" - SPOLM: Simpósio de Pesquisa Operacional e Logística da Marinha, Rio de Janeiro, 2008.

[3] ACHÃO FILHO, N. \& QUELHAS, O.L.G. - "Critérios para Aplicação de ABC (Activity Based Costing) na Indústria Naval - Revista Produção, v.13, n.1, p.91-102, São Paulo, 2003.

[4] ACHÃO FILHO, N., A simulação como método de avaliação da qualidade de atendimento hospitalar: o caso na emergência de um hospital municipal, COPPE, UFRJ, 2002.

[5] ACHÃO FILHO, N. - "Uma Proposta de Metodologia de Implementação do Sistema de Gerenciamento de Custos Baseado e Atividades ("Activity Based Management") para uma Empresa do Setor Naval ” - 160 p. (Monografia) - LATEC, UFF, 1998.

[6] CARDOSO, J.N. \& HEIN, N. - "Aplicação do Custeio Baseado em Atividade e Tempo (TDABC) em Laboratórios de Análises Clínicas - Anais SIMPOI, 2008.

[7] COGAN, Samuel - "Activity-Based Costing (“ABC"): A Poderosa Estratégia Empresarial" Rio de Janeiro - Ed. Pioneira - 1994.

[8] COOPER, R. e Kaplan, R.S. - "Custo e Desempenho: administre seus custos para ser mais competitivo" - São Paulo - Ed. Futura - 1998.

[9] COOPER, R."Implementing an Activity-Based Cost System”, Journal of Cost Management, pp.32-42, (Spring), 1990.

[10] DALMÁCIO, F. Z; REZENDE, A. J; AGUIAR, A. B. Uma Aplicação do Time-Driven ABC Model no Setor de Serviço Hospitalar: A Nova Abordagem do ABC Proposta por Kaplan e Anderson. Contab. Vista \& Rev., v. 18, n. 2, p. 11-34, 2007.

[11] DE OLIVEIRA, M.J.F.; TOSCANO, L.N.P. Emergency information support system for Brazilian public hospital, In: M.S. Rauner and K. Heidenberger (Eds.), Quantitative approaches in health care management, Peter Lang, 235-251, 2003.

[12] EVERAERT, P; BRUGGEMAN, W; De CREUS, G. Sanac Inc.: From ABC to time-driven ABC (TDABC) - An instructional case. Journal of Acc. Ed. 26, p. 118-154, 2008.

[13] KAPLAN, R.S. \& ANDERSON, S.R. - "Custeio Baseado em Atividade e Tempo", Ed. Campus, 2007.

[14] QUELHAS, O.L.G. - "Modelo de Custeio Baseado em Atividades (“ABC”) para Seleção de Fornecedores" - 151 f. - Tese (Doutorado) COPPE, UFRJ, 1994.

[15] SOUZA, A. A; AVELAR, E. A; BOINA, T. M; RAIMUNDINI, S. L. Análise da Aplicabilidade do Time-Driven Activity-Based Costing em Empresas de Produção por Encomenda. Revista Universo Contábil, FURB, Blumenau, v. 6, n.1, p. 67-84, 2010. 